\title{
Say what? Patients have poor immediate memory of major risks of interscalene block disclosed during the informed consent discussion
}

\author{
Johnny Wei Bai, ${ }^{1}$ Faraj W Abdallah (D) , ${ }^{2,3}$ Melanie Cohn, ${ }^{4}$ Stephanie Ladowski, ${ }^{1}$ \\ Poorna Madhusudan, ${ }^{1}$ Richard Brull (D) ${ }^{1,5}$
}

\begin{abstract}
Additional material is published online only. To view, please visit the journal online (http://dx.doi.org/10.1136rapm2019-100858).
\end{abstract}

'Department of Anesthesia, Toronto Western Hospital, Toronto, Ontario, Canada ${ }^{2}$ Deparment of Anesthesia, University of Ottawa Faculty of Medicine, Ottawa, Ontario, Canada

${ }^{3}$ Department of Anesthesia and the Li Ka Shing Knowledge Institute, University of Toronto, Toronto, Ontario, Canada ${ }^{4}$ Department of Psychology, University of Toronto, Toronto, Ontario, Canada

${ }^{5}$ Department of Anesthesia, Women's College Hospital Toronto, Ontario, Canada

\section{Correspondence to}

Richard Brull, Department of Anesthesia, Toronto Western Hospital, Toronto, ON M5T 2S8, Canada; richard.brull@uhn.ca

Received 19 July 2019 Revised 8 August 2019 Accepted 14 August 2019 Published Online First 23 August 2019

\begin{abstract}
Background Poor memory of disclosed risks can undermine informed consent and create medicolegal challenges. The extent to which patients remember the risks of peripheral nerve blockade following the informed consent discussion is unknown. This prospective cohort study evaluated patients' immediate memory of risks related to interscalene block (ISB) that were disclosed during the preoperative informed consent discussion. Methods Using a standardized script, patients scheduled for arthroscopic shoulder surgery were informed of the risks of ISB by an anesthesiologist in the preoperative assessment clinic. Immediately thereafter, consenting participants were asked to identify the risks of ISB from a printed list of nine true risks (four major and five minor) and nine 'distractor' items, which were unrelated adverse events and not disclosed. The primary outcome was the proportion of participants who remembered all four true major risks including longterm nerve damage, seizure, life-threatening event, and damage to the covering of the lung.
\end{abstract}

Results Among 125 participants, only 26 (21\%) remembered all four major risks of ISB. The mean number of major risks remembered was $2 \pm 1$ out of 4 . Fifteen $(12 \%)$ participants remembered all nine true risks. The mean number of true risks remembered was $6 \pm 2$ out of 9. Multivariable analysis revealed that participants' selfrated assessment of their memory was not associated with actual recall.

Conclusion Patients have poor immediate memory of the major risks related to ISB disclosed during the informed consent discussion. Under the present study conditions, the validity of the informed consent process for patients undergoing ISB may be undermined.

\section{INTRODUCTION}

Concerns related to informed consent account for over 50\% of patient complaints against anesthesiologists. ${ }^{1}$ Among these complaints, $69 \%$ are associated with inadequate disclosure of risks. ${ }^{1}$ In the setting of regional anesthesia, a significant proportion of medicolegal actions brought forth in North America and the UK pertain to issues of informed consent. $^{1-3}$ Central to the validity of informed consent is faithful understanding of the nature of the intervention, major and minor risks, alternatives, and consequences of not receiving treatment. ${ }^{4}$

For patients undergoing regional anesthesia techniques, the extent to which they understand and remember the material risks of peripheral nerve blockade (PNB) is unknown. While we have previously reported on the risks disclosed by regional anesthesia providers, there are sparse data pertaining to how these risks are received by patients. ${ }^{5}$ Current evidence of patients' memory of risks related to PNB is limited to two reports, both of which are undermined by small sample size and methodological shortcomings, including both inconsistent and incomplete risk disclosure. ${ }^{67}$ The present study aimed to determine the extent to which patients remember the risks of PNB disclosed to them by the anesthesiologist during the preoperative informed consent discussion in advance of their surgery. Specifically, we sought to identify the proportion of patients who remember the major risks related to interscalene brachial plexus block (ISB) immediately following the informed consent discussion. We hypothesized that the majority of the patients would immediately remember all major risks related to ISB that were disclosed to them during the informed consent discussion.

\section{METHODS}

\section{Study design and setting}

This single-center, prospective observational study was approved by the Women's College Hospital Research Ethics Board and conducted between August 2017 and May 2019 at Women's College Hospital, an academic ambulatory surgery hospital fully affiliated with the University of Toronto. The authors prepared this study report in accordance with the Strengthening the Reporting of Observational Studies in Epidemiology guidelines. ${ }^{8}$

\section{Study participants}

Adult patients with sports-related shoulder injuries scheduled to undergo general anesthesia for elective outpatient unilateral arthroscopic shoulder surgery, including the preoperative administration of a single-injection ISB for postoperative analgesia, were eligible to participate in this study. Patients were eligible regardless of whether or not they ultimately consented to receive an ISB for postoperative analgesia. Exclusion criteria comprised patients who were not competent for medical decision-making; non-English speaking patients; patients who refused to listen to risk disclosure in full or in part, or otherwise participate in the informed consent process; patients who had undergone ISB in the past (due to possible confounding 
recall from previous informed consent discussions); and, patients with conditions that posed special risks for ISB (eg, chronic obstructive pulmonary disease).

\section{Standard care}

As per institutional practice, patients eligible for regional anesthesia were seen by an anesthesiologist in the anesthesia preoperative assessment clinic several weeks prior to surgery, during which time the informed consent discussion and risk disclosure related to regional anesthesia took place. All eligible patients were assessed by the clinic anesthesiologist (staff anesthesiologist or anesthesia fellow), who was instructed to conduct their preoperative assessment, consultation, and planning according to their own routine practice, including discussion of the benefits and alternatives to ISB, with the exception of disclosure of the risks related to ISB.

\section{Risk disclosure}

For the purposes of this study, disclosure of risks related to ISB was standardized. At the appropriate point in time during the informed consent discussion, patients were told by the clinic anesthesiologist that the risks of ISB would be read aloud in order to ensure complete and standardized disclosure. Subsequently, the clinic anesthesiologist read aloud verbatim a pre-printed script describing the risks of ISB in simple, plain language to all eligible patients (online supplementary appendix 1). The script was largely based on the patient information pamphlet for nerve blocks of the shoulder, arm, and hand, created by the Royal College of Anesthetists in the UK. ${ }^{9}$ All eligible patients thus received the identical information regarding the major and minor risks of ISB prior to being recruited to this study.

\section{Recruitment}

On the conclusion of the preoperative assessment clinic visit with the anesthesiologist, a research coordinator approached all eligible patients for study recruitment. Patients were informed about the purpose of this study, after which written informed consent was obtained from all patients willing to participate in this study.

\section{Data collection}

A questionnaire created in conjunction with a clinical neuropsychologist and memory researcher (MC) was used to assess immediate memory of the risks disclosed during the informed consent discussion related to ISB. The questionnaire was designed to evaluate the participants' ability to correctly identify the risks of ISB that were disclosed to them in the standardized script. The questionnaire comprised 18 adverse events, including nine true risks of ISB that were disclosed to the participants and nine distractor items that were unrelated to ISB and not disclosed. Distractors items were included as control items to ensure that positive endorsement of a true risk reflected accurate memory and not merely a positive response bias (ie, endorsing all items). Among the nine true risks were four major risks (long-term nerve damage, seizure, life-threatening event, and damage to the covering of the lung) and five minor risks (temporary nerve damage, damage to a blood vessel, hoarse voice, droopy eyelid, and some difficulty breathing). The nine distractor items listed included heart attack, stroke, blood clot, damage to the covering of the heart, itchiness/rash, sore throat, swollen eyes, muscle injury, and headache. The questionnaire was scored by awarding one point for each correct selection; specifically, points were awarded for selecting 'yes' (ie, true) for true risks and selecting 'no' (ie, false) for distractor items, up to a total of 18 possible points. Additional data collected were patient demographics, including age, sex, education level, self-rated English language proficiency (Likert scale). Finally, at the end of the questionnaire, we included a subjective 5-point Likert scale self-rating of how well the participants felt they remembered the risks associated with ISB that were disclosed to them during the informed consent discussion.

\section{Outcomes}

Our clinically important primary outcome was the proportion of participants who remembered all four major risks of ISB that were disclosed during the informed consent discussion. Secondary outcomes included (i) number of major risks remembered (out of four); (ii) proportion of participants who remembered all nine true risks of ISB; (iii) number of true risks remembered (out of nine); (ii) number of distractors correctly identified (out of nine); (iii) factors independently associated with superior memory of risks. We also determined the most commonly remembered true risks and misremembered distractor items.

\section{Statistical methods}

Statistical analysis was performed using SPSS V.20. ${ }^{10}$ Descriptive characteristics and outcomes were reported as proportion $\mathrm{n}(\%)$, mean $\pm S D$, or median (IQR), depending on variable distribution. Multivariable linear regression was used to determine potential factors associated with memory of the risk discussion, using the number of risks remembered (from 0 to 9) as the outcome (dependent) variable. Predictors were chosen a priori, including age, sex, education level, self-rated English language proficiency (6-point scale from 'adequate' to 'perfect'), and participants' self-perceived ability to recognize the risks, as rated on a Likert scale (from 1 to 5). Statistical significance was defined as $\mathrm{p}<0.05$. Available-case analysis was used to report patient characteristics while complete-case analysis was used for regression.

\section{Sample size}

Sample size estimation is difficult in this observational study given the paucity and poor quality of data in this field. Based on wide estimates from previous studies on informed consent in various specialties, ${ }^{11} 12$ we assumed that at least $50 \%$ of patients would remember all major risks of ISB. In order to achieve a margin of error up of $10 \%$ with a $95 \%$ CI, we required a sample size of approximately $100 .^{12}$ To account for incomplete questionnaires, we selected a sample size of 125 .

\section{RESULTS}

A total of 187 eligible patients were approached for recruitment immediately after their informed consent discussion. Of note, 125 patients agreed to participate in this study and provided written informed consent prior to completing the study questionnaire. No participants withdrew from the study. The flow of participants is summarized in figure 1. Participant characteristics are summarized in table 1 . Among the 125 study participants, $121(96.8 \%)$ participants ultimately consented to receive an ISB for their surgery, and four (3.2\%) participants refused to receive an ISB for their surgery.

\section{Risk questionnaire scores}

Table 2 summarizes the participants' performance on the risk questionnaire. Only 26 (21\%) participants remembered all four major risks of ISB. Participants remembered a mean of $2 \pm 1$ out of the four major risks. Fifteen (12\%) participants correctly 


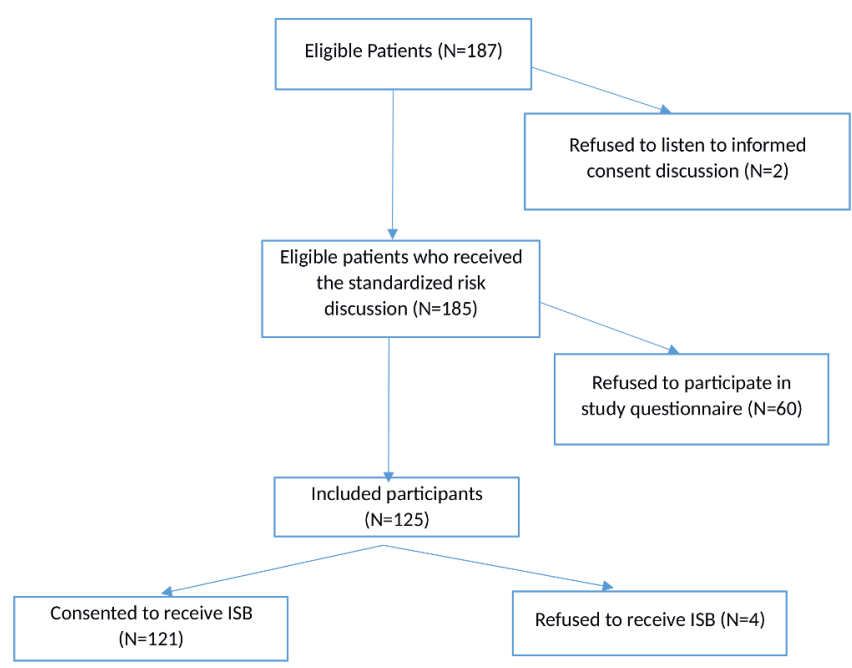

Figure 1 Participant recruitment. Flow diagram for study participants.

remembered all nine true risks of ISB disclosed during the informed consent discussion, while the mean number of true risks remembered was $6 \pm 2$ out of 9 . Participants correctly identified $6 \pm 3$ of the nine distractor items. The most commonly remembered true risks were temporary nerve damage (121 (96.8\%)) and long-term nerve damage (116 (92.8\%)). The distractors which were most commonly mistaken to be true risks of ISB were the development of a sore throat and blood clot.

\section{Factors associated with superior memory}

Multivariable linear regression was performed using the number of true risks remembered as the dependent outcome, with values ranging from 0 to 9 (table 3 ). The predictors that were selected a priori included age, sex, education level, selfrated English language ability, and self-rated ability to remember the risks disclosed (5 point-Likert scale). After adjustment for confounding, the only factor associated with increased questionnaire score was increased self-reported English proficiency, though the effect was small (adj. $\beta$ : 0.24 (95\% CI 0.05 to 0.41 ); $\mathrm{p}=0.015$ ).

\section{Self-rating of memory}

Participants were asked to rate their own memory of the disclosed risks related to ISB on a Likert-scale from 1 (very poor) to 5 (very well). The median self-rating of how well they remembered the risks was a $3.0(3.0,4.0)$, which corresponds to 'somewhat well'. In multivariable linear regression, there was no association between the participants' self-rating of their own memory of the risks disclosed and their actual risk questionnaire score (adj. $\beta$ : $0.13(95 \% \mathrm{CI}-0.21$ to 0.96$) ; \mathrm{p}=0.207)$.

\begin{tabular}{ll}
\hline Table 1 & \\
\hline Age (years) & $44 \pm 17$ \\
Females $(\mathrm{n}(\%))$ & $36(28.8)$ \\
Highest education $(\mathrm{n}(\%))$ & \\
$\quad$ Grade school & $2(1.8)$ \\
High school & $18(16.5)$ \\
College/certificate & $24(22.0)$ \\
University & $62(56.9)$ \\
\hline Postgraduate $(\mathrm{eg}, \mathrm{PhD})$ & $3(2.8)$ \\
\hline
\end{tabular}

Data presented as $n(\%)$ of participants or mean (SD). $N(\%)$ calculated from available cases.
Table 2 Proportion of participants correctly remembering each true risk and distractor

\begin{tabular}{ll}
\hline & $\begin{array}{l}\text { Patients providing correct } \\
\text { answer }(\mathbf{n}(\%))\end{array}$ \\
\hline Major risks & $116(92.8)$ \\
\hline Long-term nerve damage & $80(64.0)$ \\
\hline Damage to the covering of the lung & $65(52.0)$ \\
\hline Seizure & $38(30.4)$ \\
\hline Life-threatening event & $121(96.8)$ \\
\hline Minor risks & $96(76.8)$ \\
\hline Temporary nerve damage & $94(75.2)$ \\
\hline Droopy eyelid & $81(64.8)$ \\
\hline Damage to a blood vessel & $73(58.4)$ \\
\hline Some difficulty in breathing & \\
\hline Hoarse voice & $97(90.7)$ \\
\hline Distractors & $90(84.9)$ \\
\hline Heart attack & $89(84.0)$ \\
\hline Damage to the covering of the heart & $78(76.5)$ \\
\hline Swollen eyes & $80(76.2)$ \\
\hline Stroke & $81(74.3)$ \\
\hline Muscle injury & $75(70.8)$ \\
\hline Itchiness/rash & $70(65.4)$ \\
\hline Headache & $54(49.5)$ \\
\hline Blood clot & \\
\hline Sore throat & \\
\hline
\end{tabular}

Data presented as $n(\%)$ of participants who correctly identified each item as a true risk or distractor.

\section{DISCUSSION}

In this observational study, we found that patients have poor immediate memory of the major risks of ISB disclosed during the informed consent discussion. Overall, only one in five participants were able to remember all four major risks of ISB. On average, patients remembered only half of the four major risks disclosed, suggesting that risk disclosure under the present study conditions did not affect memory of the major risks beyond what can be attributed to mere chance or a lucky guess. Importantly, there was no association between the participants' self-assessment of their own memory and their actual memory score, which indicates that while patients believed they had understood and remembered the risks, they arguably did not.

The patients' ability to remember the risks of a procedure is distinct from yet closely related to their comprehension of the inherent risks. A patient who dutifully remembers all of the risks of a procedure may still not fully appreciate the potential adverse

Table 3 Factors associated with superior memory of risks

\begin{tabular}{llll}
\hline Predictors & $\begin{array}{l}\text { Adjusted beta } \\
\text { coefficient }\end{array}$ & P value & \multicolumn{1}{c}{$95 \% \mathrm{Cl}$} \\
\hline Age & -0.10 & 0.328 & -0.04 to 0.01 \\
Sex (female vs male) & -0.03 & 0.798 & -1.00 to 0.77 \\
Education level & 0.07 & 0.497 & -0.21 to 0.43 \\
English proficiency & 0.24 & $0.015^{*}$ & 0.05 to 0.41 \\
Self-reported memory of & 0.13 & 0.207 & -0.21 to 0.96 \\
consent discussion & & & \\
\hline
\end{tabular}

Multivariable linear regression with mean number of true risks (from 0 to 9 ) remembered as the dependent variable. Independent variables selected a priori. Self-rated English proficiency was independently associated with superior memory of risks, while self-reported memory of the consent discussion was not.

${ }^{*} \mathrm{P}<0.05$ statistically significant. 
impact of these events on their life or function. On the contrary, poor memory does not necessarily invalidate patients' understanding at the time of consent, as has been shown for patients with cognitive impairment who retain reasonable comprehension and capacity. ${ }^{13}$ Nonetheless, the ability to remember risks is a key element of understanding and remains one of the most widely accepted measures of comprehension in informed consent. ${ }^{14} 15$ Therefore, if poor memory of the risks reflects suboptimal comprehension, patients may elect to undergo a nerve block which they would have otherwise refused, or alternatively, decline a beneficial nerve block which they would have otherwise accepted, both of which arguably undermine the informed consent process. Furthermore, memory of risks may be especially important in the setting of informed consent for regional anesthesia wherein there are often significant time lapses between obtaining consent (eg, in the anesthesia preoperative assessment clinic), performing the nerve block on the day of surgery, and the development of a potential PNB-related complication. Indeed, many hospital policies and healthcare acts restrict how long a patient's consent for healthcare remains valid. ${ }^{16}{ }^{17}$ Moreover, misremembering and/or disremembering of risks can impact litigation and judgments when procedural complications arise. Patients may pursue claims of alleged substandard informed consent, and while this may be true in some instances, their allegations may reflect poor memory of a fulsome informed consent discussion rather than incomplete risk disclosure. Providers are reminded that the courts do rely on patient narratives based on their own memory of the risks disclosed, even years after the informed consent discussion and procedure took place. $^{18}$

Although patient memory of risks disclosed during the informed consent discussion has been studied in the obstetrical and general anesthesia settings, this topic has not been adequately explored in the setting of PNB. ${ }^{19-21}$ This is important as PNB carries unique and potentially serious risks, and there are often alternative anesthetic options available such as general anesthesia and systemic analgesics. Zarnegar and colleagues previously conducted a small $(n=46)$ survey to compare memory of risks related to ISB compared with those related to surgery, which were disclosed prior to shoulder arthroplasty. ${ }^{6}$ These investigators found that patients had poor memory of ISB risks, with almost half of the participants unable to identify a single risk that had been previously disclosed. However, several methodological shortcomings undermine the study's validity, including incomplete and heterogeneous risk disclosure, inconsistent documentation of the consent discussions, lack of consideration for participants' language and educational levels, small sample size, and possible recall bias due to the prolonged delay between risk disclosure and subsequent memory assessment and data collection. Burkle and colleagues also evaluated patient memory of disclosed risks related to PNB but failed to report the content of the informed consent discussion and the types of PNB discussed. ${ }^{7}$ Moreover, these authors limited their entire risk disclosure discussion to only three potential risks related to PNB. To overcome these limitations and biases, our study used a larger sample size, a standardized script, and a questionnaire that included 50\% distractor items to prevent positive response bias. We also measured and adjusted for participants' language and educational levels, as well as self-reported memory to better assess patients' perspectives of informed consent.

Our study is subject to several limitations. Most importantly, our study participants were relatively young, healthy, and educated patients with no overt cognitive impairment. As such, findings from this sample may be overly optimistic and not representative of the overall patient population receiving PNB. In addition thereto, our participants were tasked with recognizing the correct answer from a prepopulated printed list of choices rather than answering a series of open-ended questions, which facilitates success and may overestimate comprehension and memory. ${ }^{22}$ Next, our study examined only immediate memory and our results do not reliably inform delayed recall, which is most often worse. Moreover, our results may overestimate patients' memory when the informed consent discussion takes place on the day of surgery rather than days or weeks in advance as in the present study conditions. Indeed, obtaining written consent from patients for elective invasive procedures or even participation in clinical research trials on the same day as surgery is widely discouraged due to the emotional duress associated with imminent surgery that can undermine patients' capacity and voluntariness, thereby invalidating the informed consent process. ${ }^{23} 24$ Our single-center design and description of only one type of PNB (ie, ISB) may further limit the generalizability of our results. Also, since we did not directly observe the anesthesiologist in clinic, we could not determine if any unmeasured factors influenced participants' comprehension and memory of the risks disclosed. Finally, we did not investigate or address any methods that may improve patient memory of the risks disclosed. Strategies such as written or audio/visual information, extended discussions, and patient testing and feedback have all been used with reported success, ${ }^{78}$ and should be the subject of future research in the setting of informed consent for PNB. For example, studies have also shown improvement of recall with prior exposure, thus providing information about the risks and benefits of PNB in advance of the informed consent discussion (eg, distributing pamphlets in the surgeon's office) may facilitate recall. ${ }^{25}$

In summary, this observational study suggests that patients have poor immediate memory of the major risks related to ISB disclosed during the informed consent discussion. Under the present study conditions, the validity of the informed consent process for patients undergoing ISB may be undermined.

Correction notice This article has been corrected since it published Online First. The author Faraj W Abdallah's name has been corrected.

Contributors JWB reviewed the literature, managed the raw data, performed statistical analyses, and wrote the manuscript. RB reviewed the literature, planned the study, oversaw data analysis and interpretation, and wrote the manuscript. FWA, SL, and PM reviewed the literature, helped with study design, drafted the tables, and wrote the manuscript. MC designed the participant questionnaire, oversaw measurement of memory/recall, helped with interpretation of results, and wrote the manuscript. All authors had a significant role in reviewing the manuscript.

Funding FWA receives research time support from the Department of Anesthesiology and Pain Medicine and the Ottawa Hospital Research Institute, University of Ottawa, Ottawa, Ontario, Canada. RB receives research time support from the Evelyn Bateman Cara Operations Endowed Chair in Ambulatory Anesthesia and Women's Health, Women's College Hospital, Toronto, Ontario, Canada.

Competing interests None declared.

Patient consent for publication Not required.

Provenance and peer review Not commissioned; externally peer reviewed.

Data availability statement Data are available upon reasonable request. All data relevant to the study are included in the article or uploaded as supplementary information.

\section{ORCID iDs}

Faraj W Abdallah http://orcid.org/0000-0003-2435-6186

Richard Brull http://orcid.org/0000-0002-7708-8843 


\section{REFERENCES}

1. Posner KL, Severson J, Domino KB. The role of informed consent in patient complaints: reducing hidden health system costs and improving patient engagement through shared decision making. J Healthc Risk Manag 2015;35:38-45.

2. Szypula K, Ashpole KJ, Bogod D, et al. Litigation related to regional anaesthesia: an analysis of claims against the NHS in England 1995-2007*. Anaesthesia 2010;65:443-52.

3. Peng PWH, Smedstad KG. Litigation in Canada against anesthesiologists practicing regional anesthesia. A review of closed claims. Can J Anesth/J Can Anesth 2000;47:105-12.

4. Kenneth E for the CMPA. Consent: a guide for Canadian physicians. Available: https://www.cmpa-acpm.ca/en/advice-publications/handbooks/consent-a-guide-forcanadianphysicians [Accessed 20 May 2019].

5. Brull R, McCartney CJL, Chan VWS, et al. Disclosure of risks associated with regional anesthesia: a survey of academic regional anesthesiologists. Reg Anesth Pain Med 2007;32:7-11.

6. Zarnegar R, Brown MRD, Henley M, et al. Patient perceptions and recall of consent for regional anaesthesia compared with consent for surgery. J $R$ Soc Med 2015; 108:451-6.

7. Burkle CM, Njathi CW, Stoike DE, et al. Patient retention and satisfaction with information exchange concerning peripheral nerve block risks. Can J Anesth/J Can Anesth 2017;64:1075-6.

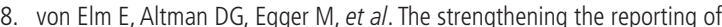
observational studies in epidemiology (STROBE) statement: guidelines for reporting observational studies. Ann Intern Med 2007;147:573-7.

9. Royal College of Anaesthetists UK. Nerve blocks for surgery on the shoulder, arm or hand:Information for patients and families. Available: https://www.rcoa.ac.uk/ document-store/nerve-blocks-surgery-the-shoulder-arm-or-hand [Accessed 20 May 2019].

10. IBM Knowledge Center. SPSS statistics. Available: https://www.ibm.com/support/ knowledgecenter/en/SSLVMB [Accessed 20 May 2019].

11. Sherlock A, Brownie S. Patients' recollection and understanding of informed consent: a literature review. ANZ J Surg 2014;84:207-10.

12. Hajian-Tilaki K. Sample size estimation in epidemiologic studies. Caspian J Intern Med 2011;2:289-98
13. Kim SYH, Karlawish JHT, Caine ED. Current state of research on decision-making competence of cognitively impaired elderly persons. Am J Geriatr Psychiatry 2002;10:151-65.

14. Kinnersley P, Phillips K, Savage K, et al. Interventions to promote informed consent for patients undergoing surgical and other invasive healthcare procedures. Cochrane Database Syst Rev 2013:40.

15. Schenker Y, Fernandez A, Sudore R, et al. Interventions to improve patient comprehension in informed consent for medical and surgical procedures. Med Decis Making 2011;31:151-73.

16. Health care (consent) and care facility (admission) act. Available: http://www.bclaws. ca/civix/document/id/consol24/consol24/00_96181_01 [Accessed 27 Jun 2019].

17. Women's College Hospital. Consent to disclose personal health information from women's College Hospital. Available: https://www.womenscollegehospital.ca/assets/ pdf/ privacy/Consent $\% 20$ to $\% 20$ Disclose $\% 20$ Personal $\% 20$ Health\%20Information\% 20FROM\%20WCH.pdf [Accessed 27 Jun 2019].

18. Boncheck LI. Informed consent: sometimes it's about memory. The Journal of Lancaster General Hospital 2017;12:65-6.

19. Paci E, Barneschi MG, Miccinesi G, et al. Informed consent and patient participation in the medical encounter: a list of questions for an informed choice about the type of anaesthesia. Eur J Anaesthesiol 1999;16:160-5.

20. Tait AR, Voepel-Lewis T, Malviya S. Do they understand? (Part I): parental consent for children participating in clinical anesthesia and surgery research. Anesthesiology 2003;98:603-8.

21. Gerancher JC, Grice SC, Dewan DM, et al. An evaluation of informed consent prior to epidural analgesia for labor and delivery. Int J Obstet Anesth 2000;9:168-73.

22. Birenbaum M, Tatsuoka KK. Open-Ended versus multiple-choice response formats - it does make a difference for diagnostic purposes. Appl Psychol Meas 1987;11:385-95.

23. Anderson OA, Wearne IMJ. Informed consent for elective surgery--what is best practice? J R Soc Med 2007;100:97-100.

24. Brull R, McCartney CJL, Chan VWS, et al. Are patients comfortable consenting to clinical anesthesia research trials on the day of surgery? Anesth Analg 2004:98:1106-10.

25. Srull TK. The role of prior knowledge in the acquisition, retention, and use of new information", in NA - advances in consumer research. In: Bagozzi RP, Tybout AM, eds. Association for consumer research. Ann Abor, MI, 1983: 10. 572-6. 